\title{
Réflexions sur l'art du mouvement : intermédialités, migration et réception de la danse
}

\section{Sarah ANTHONY, Université McGill}

Lors du colloque de l'APFUCC en 2017, dans le cadre d'un atelier organisé par Johanna Danciu et moi-même, nous avons rassemblé onze communications qui examinaient l'effet innovateur de la notion de l'inter dans des œuvres performatives. Parmi ces présentations, plus d'un tiers traitaient de l'art du mouvement. De cet heureux hasard est venue l'idée de regrouper ces études au sein d'un dossier thématique sur la danse, destiné à être publié dans Voix plurielles.

La diversité des thèmes abordés dans les travaux que recueille ce dossier, souligne à quel point la danse est un art qui permet de transmettre une pluralité de messages. Dans l'article d'Oriane Maubert, la gestuelle de style contemporain de Sans objet (2009) d'Aurélien Bory occasionne une discussion autour de l'effet produit par la rencontre intermédiale d'un robot industriel et des danseurs sur scène. Quand son corps de matière s'oppose aux corps de chair des interprètes ou lorsqu'il «fusionne » avec ces derniers, le robot devient tantôt marionnette, tantôt marionnettiste, parfois partenaire chorégraphique et souvent la source même du mouvement. En examinant ces dialogues intermédiaux, Maubert montre comment Bory renégocie l'illusion de l'animation d'un objet mécanique, parvient à recréer le geste pur évoqué par Heinrich von Kleist et renouvelle les possibilités chorégraphiques par l'hybridation des corps.

L'intermédialité fait également l'objet de mon article qui cherche à cerner comment s'effectue la transformation d'un texte en gestuelle. En prenant comme exemple La belle au bois dormant, j'étudie quatre procédés textuels - la reconfiguration, la substitution, l'élimination et l'addition - qui ont facilité la transposition du conte de Charles Perrault (1697) dans le libretto d'Ivan Vsevolozhsky (1890) pour en arriver au ballet classique de Marius Petipa (1890). À ces procédés s'ajoute l'archétype, dont l'analyse permet ensuite d'élargir mon corpus afin d'illustrer comment les chorégraphes contemporains Mats Ek et Édouard Lock utilisent celui-ci dans leurs réinterprétations du ballet de Petipa - soit Sleeping Beauty (1996) et Amjad (2007) respectivement - pour raconter une histoire par le mouvement et faire d'un classique des récits contemporains.

Dans son étude d'Ethnoscape (2015) de Cécile Proust et, en contrepoint, de Rester. Étranger (2017) de Barbara Manzetti - deux projets chorégraphiques traitant de la migration humaine - Mélanie Mesager pose des questions d'ordre éthique, à savoir si et comment la danse 
contemporaine peut présenter ou évoquer les corps des immigrés sur scène. La comparaison de ces deux œuvres accentue les façons différentes par lesquelles ces chorégraphes choisissent de s'inscrire dans le discours sur la migration - notamment par les modalités de présence des corps migrants dans leurs créations - et comment ces décisions influent sur la réception qu'on fera de leur art. À travers son analyse, Mesager découvre que ces projets chorégraphiques traitent non seulement du sujet de la migration, mais sont également des lieux de rencontre des corps.

En s'appuyant sur le concept de l'image d'Henri Bergson, le dernier article de ce dossier cherche à théoriser la réception de la danse du point de vue du spectateur et du critique. Pour ce faire, Thomas Ayouti analyse trois moments de la réception : avant le spectacle, en examinant les coordonnées du regard du spectateur, soit ses horizons d'attente et le corps même de celui-ci ; pendant le spectacle, en s'interrogeant sur le passage de l'image à la représentation ; et après le spectacle, en s'attardant sur les notions de «figure » de Mathieu Bouvier et d' « état de corps » de Philippe Guisguand, qui permettent de mieux comprendre la signification de l'œuvre chorégraphique et de verbaliser l'expérience de la danse.

Pour conclure, j'aimerais remercier la directrice de rédaction de Voix plurielles, Catherine Parayre, d'avoir accepté de publier ce dossier avec grand enthousiasme. Je suis également infiniment reconnaissante envers mes collègues - Oriane Maubert, Mélanie Mesager et Thomas Ayouti - pour leur travail remarquable et leur patiente collaboration. Aux membres du comité de lecture, j'adresse mes sincères remerciements pour leurs évaluations minutieuses des articles, qui ont certainement amélioré la qualité de ce dossier. Et pour finir, je tiens à exprimer ma profonde gratitude à l'égard de Mélanie Daigle pour son soutien et envers Johanna Danciu, qui m'a aidée à organiser l'atelier sans lequel ce dossier n'aurait pas vu le jour.

Bonne lecture ! 